\title{
Integration des Transfers in eine Theorie des Zweitspracherwerbs ${ }^{1}$
}

Heike Wiese

\begin{abstract}
Auf der Grundlage der bisherigen Forschungsergebnisse zu Transfer und Interferenz wird die Übertragung sprachlichen Wissens in die Interimsprache in eine Theorie des Zweitspracherwerbs integriert. Transfer wird dabei als interlinguale Ausprägung der Generalisierung verstanden, eine Sichtweise, die die Untersuchungsergebnisse zu Bedingungen und Ursachen des Transfers in einen Erklärungszusammenhang mit anderen Phänomenen des Zweitspracherwerbs stellt. Darüberhinaus ermöglicht es diese Interpretation, eine Verbindung zu kognitiven Leistungen des Lerners beim Erstspracherwerb sowie beim Lernen überhaupt herzustellen.
\end{abstract}

\section{Stand der Forschung zum Transfer im Zweitspracherwerb}

Das Interesse an der Interferenz im ZSE konzentriert sich im wesentlichen auf drei Fragestellungen: Welchen Einfluß hat die Interferenz, wann tritt sie auf, und wie wirkt sie sich - gemeinsam mit anderen muttersprachlichen Einflüssen - auf den Spracherwerb aus?

Während anfangs die Diskussion vor allem darüber geführt wurde, ob die Interferenz im Zweitspracherwerb eine Rolle spielt und wie groß ihr Stellenwert ist, fokussieren neuere Untersuchungen stärker die Bedingungen und Ursachen des Transfers. Darüberhinaus richtet sich das Interesse auch auf weitere Auswirkungen der L1 beim Zweitspracherwerb, wie etwa die Vermeidung bestimmter Strukturen und andere L1-bedingte Normverstöße, die nicht als Interferenzen im engeren Sinne anzusehen sind (vgl. etwa Dulay/Burt/Krashen 1982; Beardsmore 1986; Czochralski 1973).

\subsection{Definitionen zu Transfer und Interferenz}

\footnotetext{
${ }^{1}$ Dies ist die schriftliche und in einigen Punkten veränderte Fassung eines Vortrags auf dem nationalen Deutschlehrer-Kongreß der Goethe-Institute Athen / Thessaloniki, April 1991.
} 
Da die Termini "Transfer" und "Interferenz" bislang nicht einheitlich verwendet Fehler werden, gebe ich an dieser Stelle einen Überblick über diejenigen Definitionen, die ! den meisten Theorien zugrundeliegen.

"Kleinster gemeinsamer Nenner" aller Definitionen ist die Charakterisierung des nicht Transfers (bzw. der Interferenz) als Verwendung von Einheiten oder Strukturmustern ert. einer Sprache in einer anderen bzw. als Übertragung von Einheiten oder Strukturmustern einer Sprache in eine andere als Folge von Sprachkontakt.

Die verschiedenen Ansätze unterscheiden sich dann durch ihre Spezifikation des Transfers/der Interferenz nach folgenden Gesichtspunkten:

I. Transfer/Interferenz als Vorgang oder als Ergebnis der Übertragung;

II. Übertragung/Verwendung von Einheiten oder von Strukturmustern;

III. Transfer/Interferenz als Begriff der parole oder Begriff der langue;

IV. Konsistenz oder Inkonsistenz der Übertragung/Verwendung in Bezug auf den jeweiligen Sprecher;

V. Übertragung, die zu einem Normverstoß oder zur Einhaltung der Norm führt.

Durch die Gegenüberstellung der Termini "Transfer" und "Interferenz" wird meist zwischen Vorgang und Ergebnis der Übertragung muttersprachlicher Elemente unterschieden. Diese Unterscheidung ist jedoch weitgehend auf Arbeiten der 50er und 60er Jahre beschränkt (z.B. Haugen 1956; Weinreich 1963; Mackey 1976), ebenso wie der Gebrauch unterschiedlicher Begriffe für die Übertragung von L1-Einheiten und die von L1-Strukturmustern in die L2.

Häufig wird hier eine Differenzierung zwischen Phänomenen der parole ("Interferenz") und solchen der langue ("Integration") vollzogen (z.B. Haugen 1956; Mackey 1976; Juhász 1982). Später gehen viele Ansätze von einer mischsprachlichen Kompetenz aus (z.B. Oksaar 1979; Clyne 1975) und treffen eine Unterscheidung eher nach Konsistenzkriterien (LePage 1969). Vor allem im Bereich der Bilingualismusforschung setzt sich eine neue Auffassung von Norm durch, die auch zweisprachige Gemeinschaften einbezieht.

Damit ändert sich auch die Definition der Normabweichung bzw. -einhaltung, die nun in Bezug auf die Konsistenz des Sprachgebrauchs (etwa bei LePage 1969) oder auf die Akzeptanz der Äußerung in bilingualen Gemeinschaften (wie bei Beardsmore 1986) formuliert wird, während "Norm" im Bereich der Zweitspracherwerbsforschung meist für einsprachige Gemeinschaften definiert ist (z.B. bei Juhász 1970). Norm manifestiert sich hier in der Akzeptanz von Äußerungen in einer weitgehend monolingualen Umgebung. Eine Abweichung durch ein einzelnes Individuum - auch wenn sie innerhalb seines Sprachgebrauchs konsistent auftritt - schafft nach dieser Auffassung nicht neue Normen, sondern stellt in erster Linie einen Verstoß gegen die bestehenden dar. 
Für die muttersprachlich bedingte Einhaltung der Norm im Fremdspracherwerb wer- ${ }^{\text {Fehler }}$ den z.T eigene Begriffe eingeführt (etwa Juhász 1970).

Das Schaubild unter Abb. (1) gibt einen - wenn auch stark vereinfachenden - nicht Überblick über ähnliche Definitionen in den verschiedenen Ansätzen.

\section{[hier Abbildung 1]}

\subsection{Bedingungen und Ursachen für das Auftreten von Interferenzen}

Die Ursachen für Interferenzen und die Bedingungen, unter denen sie auftreten können, wurden hauptsächlich seit Beginn der 80er Jahre und in erster Linie von Vertretern der "integral-part-Hypothese" untersucht. Diese integriert den Transfer in den ZSE, indem sie ihn als kognitiven Prozeß begreift, der beim ZSE neben universellen Spracherwerbsmechanismen wirksam werde (etwa Wode 1988; Jansen/Lallemann/Muysken 1981; Andersen 1983).

Als Ergebnis der verschiedenen Arbeiten kristallisieren sich fünf Faktoren heraus, die Transfer begünstigen:

I. "Kritische Ähnlichkeit" zwischen den jeweiligen Elementen der involvierten Sprachen

Die Tatsache, daß Elemente, die als ähnlich empfunden werden, eher Interferenzen verursachen als solche, zwischen denen ein scharfer Kontrast besteht, konstatiert bereits Juhász (1970), wenn er die These aufstellt, "Kontrast-Mangel" (Juhász 1970:135) führe am ehesten zu Interferenzen. Er bezieht sich dabei auf die experimentelle Psychologie, in der die "homogene Hemmung" (Juhász 1970:97) als Ranschburgsches Phänomen bekannt ist.

Von Vertretern der "integral-part-Hypothese" wird diese Auffassung insoweit übernommen, als die Ähnlichkeit der Elemente als einer von mehreren Faktoren, die zu Interferenzen führen, angesehen wird. So postuliert etwa Wode (1985:25), es müsse "eine kritische Ähnlichkeit zwischen den Elementen der neuen Zielsprache und der zuvor gelernten Sprache(n) bestehen, damit sie füreinander susbstituiert werden können".

\section{I Subjektiv wahrgenommene geringe Distanz zwischen L1 und L2}

Kellerman (1983) bezeichnet den Sachverhalt, daß der Lerner die linguistischen Systeme der involvierten Sprachen als ähnlich wahrnimmt, als eine Voraussetzung für Interferenzen. Muttersprachlicher Transfer trete vor allem dann auf, wenn die Distanz zwischen L1 und L2 als gering empfunden werde. Entscheidend ist hierbei vermutlich 
nicht unbedingt die tatsächliche strukturelle Ähnlichkeit der Sprachen, sondern viel-Fehler mehr die diesbezüglichen Intuitionen der Lerner.

\section{I . L2-Entwicklungsstand des Lerners}

und L2 oder die "kritische Ähnlichkeit" einiger ihrer Strukturen wahrzunehmen, von seinem L2-Entwicklungsstand abhängig. Lerner transferieren nach dieser These nur dann, wenn sie in Bezug auf eine bestimmte Struktur eine Stufe in ihrer L2-Entwicklung erreicht haben, die es ihnen erlaube, die teilweise Übereinstimmung dieser Struktur mit einer L1-Struktur zu erkennen.

\section{Markiertheit des L2-Elements}

Die stärkere Markiertheit des L2-Elements gegenüber dem L1-Element wird in den meisten Ansätzen als eine Voraussetzung für Transfer angesehen. Im Bereich der Interferenzforschung im ZSE hat sich hierbei eine Definition zur Markiertheit durchgesetzt, die auf Eckmans (1977) Formulierung zurückgeht:

"A phenomenon A in some language is more marked than $\mathrm{B}$ if the presence of $\mathrm{A}$ in a language implies the presence of $\mathrm{B}$; but the presence of $\mathrm{B}$ does not imply the presence of A." (1977:320)

Er postuliert in seiner "Markedness Differential Hypothesis", beim ZSE verursachten diejenigen Bereiche Schwierigkeiten, die nach universalgrammatischen Gesichtspunkten markiert seien. Transfer trete dann am ehesten auf, wenn die muttersprachlichen Strukturen weniger markiert, somit einfacher seien, und daher auch aufgrund universeller Erwerbsschemata bevorzugt würden.

\section{Inkonsistenz der L2}

Während einige Arbeiten davon ausgehen, daß oft sowohl negativer Transfer als auch die größere Komplexität oder Inkonsistenz der L2 für Fehler verantwortlich seien², stellen andere einen Zusammenhang zwischen den beiden Phänomenen her und fassen Abweichungen innerhalb des L2-Systems als Stimulus für Interferenzen auf ${ }^{3}$. Interferenzen stellen nach dieser Theorie Strukturen dar, die typologisch möglich sind; sie sind - in Anlehnung an Coseriu (1973) - Abweichungen von der Norm, nicht jedoch Verstöße gegen das System.

Als Ergebnis der verschiedenen Untersuchungen läßt sich somit festhalten, daß Transfer nur dann stattfindet, wenn zwischen den betreffenden Elementen von L1 und L2 eine

\footnotetext{
${ }^{2}$ z.B. Adiv (1984)

${ }^{3}$ z.B. Andersen (1983)
} 
"kritische Ähnlichkeit" besteht, d.h. wenn eine partielle Übereinstimmung vorliegt, Fehler die dem Lerner als Kongruenz erscheint. Die Ähnlichkeit wird umso eher als ! Gleichheit wahrgenommen, je geringer dem Lerner die Distanz zwischen L1 und L2 Textm erscheint. Voraussetzung für diese Lernerperzeption ist ein "entwicklungsspezifisch- nicht defini chronologisches Moment" (Wode 1985:25): Der Lerner müsse einen Entwicklungs- ert. stand erreicht haben, der ihm erlaube, die Übereinstimmungen einer L2- mit einer L1Struktur zu erkennen. Als Stimuli für Transfer wirken - in Übereinstimmung mit universellen Spracherwerbsmechanismen - die Markiertheit des betreffenden L2-Elements sowie die Inkonsistenz der L2 in dem entsprechenden Bereich.

\subsection{Auswirkungen der L1 im Zweitspracherwerb}

Der Einfluß der Muttersprache im ZSE führt nicht nur zu Interferenzen, sondern äußert sich außerdem in verschiedenen anderen Formen: Einerseits kann der Rückgriff auf die L1 einen Einfluß auf die Erwerbsgeschwindigkeit haben, andererseits können Unterschiede zwischen Muttersprache und Zielsprache zu Schwierigkeiten führen, ohne jedoch Interferenzfehler zu verursachen. Zu den Auswirkungen der L1 gehören nach dem derzeitigen Forschungsstand folgende Phänomene:

I. Interferenzen bei kritischer Ähnlichkeit von L1- und L2-Strukturen;

II. Vermeidung abweichender Strukturen;

III. Simplifikation und andere Normverstöße bei typologisch stark divergierenden Strukturen;

IV. Beschleunigung oder Verzögerung des Erwerbstempos, wenn Strukturen der L1 universellen Erwerbsstufen ähneln.

Bei der Untersuchung von Interimsprachen in Bezug auf muttersprachliche Einflüsse muß somit stets berücksichtigt werden, daß der Rückgriff auf die L1 nicht nur in Interferenzen resultiert, sondern darüberhinaus die Ursache verschiedener weiterer Erscheinungen sein kann.

Aufgrund solcher Überlegungen werden in einigen Ansätzen die Termini "Transfer" und "Interferenz" zugunsten des umfassenderen Begriffs "cross-linguistic influence" aufgegeben (vgl. Kellerman 1983), der alle Auswirkungen der L1 bezeichnen soll.

\subsection{Zum Status des Transfers in der Forschung}

In den meisten Theorien zum ZSE wird Transfer separat behandelt und kaum in Bezug zu den anderen Phänomenen des Spracherwerbs gesetzt. Transfer wird meist entweder als isoliert auftretendes, rein zweitspracherwerbsspezifisches Phänomen betrachtet, das im ESE kein Pendant besitzt (z.B. bei Keim 1984; Andersen 1983), oder als eigenständige, zusätzliche Strategie des ZSE angesehen (etwa bei Clahsen/Meisel/Pienemann 1983; Wode 1988), ohne daß dieser Status jedoch hinreichend 
begründet und ein Zusammenhang zu den anderen, universellen Spra-Fehler cherwerbsstrategien hergestellt wird.

2. Der Status von Transfer und Interferenz in einer Theorie des nicht Zweitspracherwerbs

ert.

Ich werde im folgenden einige Thesen zu Lernstrategien im Spracherwerb entwerfen und vor diesem Hintergrund den Transfer in eine Theorie des ZSE integrieren. Ich werde hierzu den Zusammenhang, der zwischen den zentralen universellen Spracherwerbsstrategien besteht, verdeutlichen und auf dieser Grundlage aufzeigen, wo Transfer als spezifische Ausprägung dieser Strategien im Rahmen des ZSE wirksam werden kann. Eine solche Theorie soll darüberhinaus die Bedingungen, unter denen Transfer auftritt, erhellen und so zur Erklärung der in 1.2. zusammengefaßten empirischen Befunde beitragen. 
Nachdem ich mich bis jetzt auf die vorläufige Charakterisierung aus 1.1. bezogen ! habe, ist es für den Fortgang der Argumentation notwendig, in Bezug auf Transfer arke und Interferenz eine detailliertere Definition zu formulieren, die verschiedenen nicht weiteren bislang dargestellten Aspekten Rechnung trägt und das Phänomen des efini Transfers in einen Theorierahmen stellt, der die Grundlage für meine weiteren Überlegungen bilden soll.

\section{DEFINITION:}

Bei der Ausbildung von Interimsprachen im ZSE liege Transfer vor genau dann, wenn der Lerner sprachliches Wissen aus zuvor erworbenen Sprachen verwendet.

Führt diese Übertragung zu einer Abweichung von der zielsprachlichen Norm, so heiße das Produkt dieses Prozesses Interferenz.

Diese Definition enthält folgende Implikationen:

Erstens beziehe ich in Abhebung von der ursprünglichen Definition die Übertragung sämtlicher sprachlicher Techniken ein und beschränke Transfer nicht auf die Verwendung linguistischer Strukturmuster und Einheiten im engeren Sinne. Dies halte ich nach Ergebnissen von Arbeiten aus dem Bereich der "integral-part-Hypothese", die Interferenzen auch in Bezug auf Pragmatik, Prosodie und grundlegende sprachliche Techniken konstatieren, für sinnvoll.

Zweitens stelle ich Transfer als Spezifikum des Zweitspracherwerbs dar, eine Eingrenzung, die ich hier aus rein pragmatischen Erwägungen vornehme, da meine Überlegungen primär den ZSE betreffen. Transfer als Charakteristikum von Lernen im allgemeinen werde ich in 2.4. kurz gesondert behandeln.

Drittens definiere ich Transfer auf der Grundlage der Interimsprachentheorie, beziehe mich daher vorwiegend auf Thesen der mentalist theory (etwa Wode 1988), die ich wegen ihrer kognitiven Basis in Bezug auf Spracherwerb für angemessener als etwa behavioristische Theorien halte.

Viertens umfaßt Transfer hier nur den proaktiven Transfer; der Terminus schließt eine retroaktive Übertragung aus. Das soll nicht bedeuten, daß eine solche beim ZSE nicht vorkommt, sondern lediglich, daß sich meine Überlegungen auf die Übertragung von zuvor erworbenen auf später gelernte Sprachen konzentrieren werden. Hierbei sind mit "zuvor erworbenen" nicht nur Sprachen gemeint, die der Lerner wie seine Muttersprache oder zumindest relativ gut beherrscht, sondern auch solche, die er nur bruchstückhaft kennt. 
Fünftens differenziere ich zwischen Vorgang und Ergebnis negativer Übertragung. Fehler Dies ist deshalb sinnvoll, weil dem Transfer als kognitivem Prozeß ein Status im Be- ! reich der Spracherwerbsstrategien zukommt, während Interferenzen linguistische Textm Entitäten im weiteren Sinne darstellen, die als Normabweichungen etwa im Rahmen nicht Entitaten im weiteren Sinne darstellen, die als Normabweichungen etwa im Rahmen nt einer Fehleranalyse konstatierbar sind.

ert.

"Norm" sei hierbei in Anlehnung an Coseriu als "die formalisierte Gesamtheit der traditionellen Realisierungen" des Systems (Coseriu 1973:44) definiert. Ich beziehe mich mit dieser Definition - in Übereinstimmung etwa auch mit Juhász (1985) ${ }^{4}$ - primär auf die Normen in im weitesten Sinne monolingualen Gemeinschaften, wobei die tatsächlich gesprochenen Varianten der Standardsprache - Soziolekte, Dialekte etc. Grundlage der kommunikativ-sozialen Akzeptanz von Äußerungen seien. Eine solche Explikation erlaubt es am ehesten, interimsprachliche Elemente in Bezug zur Zielsprache zu setzen, und ist so für die Zweitspracherwerbsforschung besonders geeignet.

\subsection{Lernstrategien im Spracherwerb}

Ich gehe für den (Erst- und Zweit-) Spracherwerb von denselben grundlegenden Entwicklungsprinzipien wie die Vertreter der mentalist theory aus und sehe als kognitive Leistung des Lerners die Dekomposition und Rekonstruktion der Sprache an: Nach dem Prinzip der "creative construction" baut er ein (unbewußtes) Regelsystem auf, das dazu beiträgt, den Lernstoff zu verringern. Äußerungen des Inputs werden nicht als Ganzes gelernt, sondern zerlegt und selektiv rezipiert. Auf der Grundlage seines bisherigen Wissens stellt der Lerner Hypothesen über das System der Zielsprache auf, die er dann im Output testet. Das kreative Vorgehen des Lerners im Spracherwerb umfaßt also einerseits die Analyse des Inputs und das Herausfiltern bestimmter - für die jeweilige Entwicklungsstufe spezifischer - Informationen (Dekomposition) und andererseits die Reintegration sprachlicher Elemente beim Aufbau der Interimsprache (Rekonstruktion).

Als zentrale Lernerstrategie sehe ich hierfür die Simplifizierung an. Sie tritt in zwei Formen auf: als Simplifizierung der Struktur des sprachlichen Systems und als Simplifizierung der Struktur seiner einzelnen Elemente. Den ersten Fall nenne ich Generalisierung, den zweiten Simplifizierung im engeren Sinne.

Bei der Generalisierung überträgt der Lerner sprachliche Elemente von bereits bekannten in neue Kontexte. Durch diese Verallgemeinerung bezüglich ihrer Verwendung wird die Anzahl der Elemente geringer gehalten, das System regularisiert und seine

\footnotetext{
4 vgl. Juhász (1985:70): "Unter Norm ist [...] systemlinguistisch gesehen eine im Prinzip sozial sanktionierte Realisierung des potentiell im System Gegebenen, soziolinguistisch gesehen eine nicht nur im Prinzip sozial sanktionierte, sondern auch situativ determinierte Verwendung sprachlicher Mittel zu verstehen."
} 
Struktur somit einfacher. Dieser Simplifizierung auf Systemebene steht die Sim-Fehler plifizierung im engeren Sinne als strukturelle Reduzierung der einzelnen ! sprachlichen Elemente gegenüber.

Sowohl Generalisierung als auch Simplifizierung i.e.S. werden einerseits in Bezug nicht auf Einheiten und andererseits in Bezug auf Strukturmuster wirksam, wobei diese ert. nicht auf linguistische Strukturen im engeren Sinne beschränkt sind, sondern sich ebenso auf pragmatische, das Textwissen betreffende, Diskurs- und andere sprachliche Techniken beziehen (vgl. 2.1.).

Die Generalisierung führt als Verallgemeinerung über das Auftreten bereits bekannter Strukturmuster zu einer Simplifizierung im Bereich des Regelinventars, als Verallgemeinerung bezüglich der Verwendung von Einheiten zu einer Simplifizierung im Bereich des Einheiteninventars. Grundlage für die Generalisierung ist die Hypothese, die Elemente, die übertragen werden, seien mit dem neuen Kontext kompatibel. Diese Hypothese basiert auf der Annahme, die zielsprachlichen Elemente seien in beiden Kontexten die gleichen. Gestützt wird diese Annahme durch die Wahrnehmung des Lerners, die Kontexte seien ähnlich, die Elemente somit eher übertragbar. Diese subjektive Übertragbarkeit der Elemente setzt außerdem voraus, daß der Lerner sie nicht als spezifisch für einen bestimmten Kontext ansieht, wie dies etwa bei Einheiten in idiomatischen Wendungen der Fall sein kann. Die Möglichkeit, Übereinstimmungen sowohl zwischen Elementen als auch ihren Kontexten wahrzunehmen, ist dabei vom Entwicklungsstand des Lerners abhängig: Erst wenn er in Bezug auf die Elemente und auf die Sprache insgesamt ein bestimmtes Wissen erworben hat, kann er Ähnlichkeiten erkennen.

Voraussetzung für die Generalisierung ist also die subjektive Ähnlichkeit der Elemente, basierend auf einem bestimmten Entwicklungsstand des Lerners und gestützt durch seine Wahrnehmung der betreffenden Kontexte als ähnlich und der Elemente als übertragbar. Diese Lernerstrategie kann zu Abweichungen von der zielsprachlichen Norm durch Übergeneralisierung führen, i. e. durch die Übertragung von Regeln oder Einheiten in Kontexte, in die dies nicht möglich ist.

Die Simplifizierung i.e.S. bewirkt durch die strukturelle Reduzierung von Strukturmustern eine Simplifizierung im Bereich der Regelanwendung, durch die strukturelle Reduzierung von Einheiten eine Simplifizierung im Bereich der Verwendung und Rezeption von Einheiten. In Bezug auf die Zielsprache kann dies zu Normverstößen durch Übersimplifizierung führen.

Simplifizierung i.e.S. und Generalisierung werden nicht nur im Bereich des Outputs wirksam, sondern betreffen schon die Verarbeitung des Inputs: Zielsprachliche Äußerungen werden - besonders zu Beginn des jeweiligen Lernvorgangs - bereits vereinfacht 
rezipiert. Sowohl bei der Rezeption als auch bei der Produktion wirken Gene-Fehler ralisierung und Simplifizierung i.e.S. zusammen. Diese Interaktion führt dazu, daß $\frac{\text { ! }}{\text { Textm }}$ der Lerner eher bezüglich der Verwendung solcher Strukturmuster und Einheiten arke verallgemeinert, die auch aufgrund der Simplifizierung i.e.S. bevorzugt werden. Die nicht Einfachheit der Elemente wirkt so als Stimulus für die Generalisierung.

Das Verhältnis, in dem die bislang besprochenen Phänomene zueinander stehen, läßt sich schematisch verdeutlichen; vgl. Abb. (2):

[hier Abbildung 2]

\subsection{Transfer als spezifische Ausprägung der Generalisierung im Zweitspracherwerb}

Während sich die bisherigen Ausführungen sowohl auf den Erst- als auch auf den Zweitspracherwerb bezogen und somit universelle Spracherwerbsphänomene betrafen, kann eine Beschäftigung mit dem Transfer nach meiner obigen Definition (2.1.) nur im Zusammenhang mit dem ZSE stattfinden. Dies bedeutet jedoch nicht, daß dem Transfer im Rahmen meiner überlegungen der Status eines Charakteristikums des ZSE zukommt, das mit den Phänomenen des ESE nichts gemeinsam hat. Ich sehe Transfer nicht als separate, zusätzliche Lernstrategie des ZSE an, sondern als eine - zweitspracherwerbsspezifische - Ausprägung der Generalisierung: Diese umfaßt demnach nicht nur die intralinguale Übertragung, sondern auch die Übertragung von Regeln oder Einheiten aus früher erworbenen Sprachen in die L2.

Transfer erhält so den Status einer interlingualen Komponente der Generalisierung und unterliegt als solche denselben Regularitäten, die auch universell für die Generalisierung gelten. Ich werde daher meine Kategorisierung im folgenden rechtfertigen, indem ich Bezug auf die oben (in 2.2.) dargestellten Charakteristika der Generalisierung im allgemeinen nehme und ihre spezielle Ausprägung beim Transfer in Anlehnung an die Forschungsergebnisse aus dem ersten Abschnitt erkläre.

Wie die Generalisierung im allgemeinen beinhaltet der Transfer die Übertragung von Elementen in neue Kontexte - d.h. hier in die L2. Auch dieser Vorgang basiert auf der Lernerhypothese, die Elemente seien mit dem neuen Kontext kompatibel. Als grundlegende Voraussetzung muß daher auch beim Transfer die These des Lerners angesehen werden, die Einheiten und Strukturmuster, die er zum Gegenstand der Übertragung macht, seien in beiden Kontexten - in diesem Fall in L1 und L2 - die gleichen. Diese Annahme stimmt mit dem oben (1.2.) vorgestellten Konzept der kritischen Ähnlichkeit überein: Bei sehr ähnlichen Elementen erkennt der Lerner mitunter nicht, 
daß die Übereinstimmung nur partiell ist; die Elemente werden als identisch Fehler $_{\text {I }}$ wahrgenommen und übertragen.

Dies kann darüberhinaus zu einer wie in 1.3. besprochenen Verzögerung des Spra- Textm cherwerbs führen: Durch die große psychische Ähnlichkeit der in die Interimsprache nicht übertragenen Elemente der L1 mit den entsprechenden der L2 kann der Lerner nur ert. schwer zwischen interim- und zielsprachlichen Strukturen differenzieren, hat dadurch größere Schwierigkeiten, die abweichende Form zu ersetzen, als Lerner anderer Muttersprachen und bleibt länger auf einer bestimmten Entwicklungsstufe stehen.

Als Stimulus für den Transfer wirkt die Einschätzung des Lerners, das Auftreten der betreffenden L1-Elemente sei nicht auf einen einzigen Kontext beschränkt, sie seien vielmehr sprachneutral und nicht sprachspezifisch, träten also auch in anderen Systemen als dem der L1 auf.

Strukturmuster und Einheiten werden umso eher als übertragbar angesehen, je ähnlicher sich auch die Kontexte dem Lerner darstellen; die subjektive Wahrnehmung des Lerners, die involvierten Sprachen seien ähnlich, stützt somit seine Hypothese. Diese Lernerperzeption - für die Kellerman (1983:114) den Begriff "psychotypology" prägte muß nicht unbedingt strukturelle Ursachen haben. So übertragen Lerner mitunter beim Erlernen einer Fremdsprache L2 eher Elemente aus einer anderen, nur bruchstückhaft beherrschten Fremdsprache L3 in die Interimsprache als aus ihrer Muttersprache, ohne daß die L3 der L2 strukturell näherstünde als die L1. Tertium comparationis ist hier möglicherweise die mangelhafte Beherrschung der Sprachen, die sich dadurch dem Lerner ähnlich präsentieren.

Diesen Lernerwahrnehmungen kommt hier im Vergleich zur intralingualen Generalisierung vermutlich noch ein größeres Gewicht zu, da davon auszugehen ist, daß Lerner eher dazu neigen, innerhalb eines sprachlichen Systems zu generalisieren, als über Systemgrenzen hinweg. Die subjektive Ähnlichkeit der Kontexte und die Einschätzung der Elemente als kontextneutral ist daher wohl bei interlingualer Generalisierung von noch größerer Relevanz.

Wie schon in 2.2. allgemein für die Generalisierung besprochen, ist auch beim Transfer ein bestimmter Entwicklungsstand Bedingung für die hier aufgeführten Lernerperzeptionen: Um einerseits die "kritische Ähnlichkeit" der für einander substituierten Elemente und andererseits die Ähnlichkeit der beiden Sprachen und die Sprachneutralität der Elemente wahrnehmen zu können, muß der Lerner sowohl in Bezug auf die L2 als auch in der L1 einen Entwicklungsstand erreicht haben, der es ihm erlaubt, Übereinstimmungen zu erkennen.

Wie bei der interlingualen Generalisierung besteht auch beim Transfer eine Wechselbeziehung zur Simplifizierung i.e.S.: Die Einfachheit von L1-Elementen wirkt als Stimulus für ihre Übertragung in die Interimsprache. Interferenzanfällig sind daher 
primär die Bereiche, in denen die L2 komplexer ist als die L1. Dies ist einerseits der Fehler Fall, wenn die L2-Struktur in einem Bereich inkonsistent ist, in dem die Übertragung ! aus der L1 zur Regularisierung führen würde, und andererseits, wenn ein L2-Element arke stärker markiert ist als das entsprechende L1-Element - auch hier also eine Überein- nicht stimmung mit den Ergebnissen der Interferenzforschung. Markiertheit ist dabei wie ert. in 1.2. im Sinne von Eckman (1977) zu verstehen: Diese Theorie, die in weiten Bereichen zu gleichen Bewertungen wie die der Prager Schule kommt und die Grundlage für Aussagen bildet, wie sie etwa Mayerthaler (1981) in seinem Ansatz macht, bietet durch ihre vorwiegend formale Orientierung und ihre interlinguale Formulierung die günstigsten Voraussetzungen für Untersuchungen im Bereich der Interferenzforschung. Abschließend ist festzustellen, daß die hier vorgenommene Integration des Transfers in den ZSE die Aussagen der "integral part"-Hypothese sowohl im Hinblick auf den Stellenwert des Transfers als auch, was die Ursachen von Interferenzen angeht, stützt: Die These, Transfer sei ein integrierter Bestandteil des ZSE, gewinnt durch seine Darstellung als Ausprägung der universellen Lernerstrategie der Generalisierung noch an Deutlichkeit. Diese Charakterisierung bindet den Transfer darüberhinaus in den Spracherwerb im allgemeinen ein, ein Ansatz, der so die Integration des Transfers in die Prozesse, die die Ausbildung von Interimsprachen im ZSE steuern, expliziert. Die Folgerungen, die sich aus den hier entworfenen Thesen ergeben, stimmen - wie jeweils dargestellt - mit den Forschungsergebnissen aus dem ersten Abschnitt überein; die hier vorgenommene Integration stellt daher die Aussagen über Ursachen und Bedingungen des Transfers in einen Erklärungszusammenhang mit universellen Phänomenen des Spracherwerbs und erhellt so ihren Status.

Um den Zusammenhang zwischen den allgemeinen Charakteristika der Generalisierung und ihren interlingualen Ausprägungen beim Transfer noch einmal hervorzuheben und so meine oben vorgenommene Kategorisierung zu unterstreichen, stelle ich die beiden Formen in der folgenden Tabelle gegenüber (Abb.3):

[hier Abbildung 3] 
Transfer tritt nicht nur im ZSE auf, sondern ist auch als allgemeines Charakteri- ${ }_{\text {Textm }}$ ! stikum des Lernens bekannt. Ansätze, die sich mit diesem Phänomen beschäftigen, arke stützen meine Überlegungen in verschiedenen Bereichen. Ich werde in diesem Ab- nicht schnitt zur besseren Unterscheidung der beiden Erscheinungen Transfer $a$ ert. verwenden, wenn ich mich auf den Transfer im allgemeinen beziehe, während der Terminus Transfer ZSE meiner obigen Definition entspreche, die eine Übertragung nur im Rahmen des ZSE beschreibt.

Transfer $_{a}$ wird in der Lernpsychologie ganz allgemein als die "Einwirkung vorangegangener Lernleistung auf eine nachfolgende" (Drever/Fröhlich 1974:283) definiert. Ebenso wie in der Zweitspracherwerbsforschung wird hierbei zwischen negativem und positivem Transfer ${ }_{a}$ unterschieden, wobei sich das Hauptaugenmerk jedoch auf letzteren richtet. Im Gegensatz zu den meisten Ansätzen aus dem Bereich des ZSE zeichnet sich die Pädagogische Psychologie so durch eine vorwiegend positive Einschätzung des Transfers $a$ aus.

Transfer $_{\mathrm{a}}$ wird hier nicht als eigene Lernstrategie aufgefaßt, sondern stellt als Sonderform von Lernen eine kognitive Leistung dar, die die Voraussetzung für die Anwendung einmal erworbenen Wissens in neuen Situationen bildet.

Transfer $_{a}$ als Anwendung des Gelernten unter neuen Bedingungen wird so aus pädagogischer Sicht angestrebt und unterstützt.

Diese im Bereich des ZSE bislang weitgehend vernachlässigte Sichtweise wird in der detaillierteren Definition des Transfers a deutlich, die Schiefele/Krapp (1981) formulieren:

"Transfer ist ein interaktionaler Prozeß, in dem unter interferenter Beteiligung von Ich-Kräften und Umwelt-Faktoren (personal-kontextliche Bedingungen eines Transfers) eine Umsetzung und Anwendung von Gelerntem in ähnlichen oder neuen Situationen erfolgt: das ist nur möglich, wenn definierbare Übereinstimmungen zwischen Lern- und Anwendungssituation bestehen (sachstrukturale Bedingungen eines Transfers)." (S.384)

Diese Definition erhellt einige Aspekte des TransfersZSE im ZSE: So sind die oben besprochenen Ursachen für Interferenzen den sachstrukturalen Bedingungen des Transfers zuzuordnen, während in den personal-kontextlichen Bedingungen einer der Gründe für die Schwierigkeiten bei der Voraussage von TransferzSE zu sehen ist.

Die Psychologie unterscheidet zwischen vertikalem Transfer $a$ als der Übertragung des Gelernten von einfachen in komplexe Bereiche und lateralem Transfer $_{a}$ als der breiten Anwendung eines bestimmten Kenntnisniveaus (vgl. Gagné 1969). Vertikaler Transfer muß nach dieser Terminologie auch für den Spracherwerb als grundlegende Voraussetzung für das Fortschreiten auf höhere Entwicklungsstufen angenommen werden, 
während im Bereich der interlingualen Generalisierung meist lateraler Transfer a auf- Fehler tritt, der Interferenzen bewirken kann. In diesem Fall bestünde dann die Aufgabe des ! Lerners beim ZSE im Diskriminationslernen, das die Differenzierung zwischen L1- Textm und L2-Elementen zum Ziel hat.

Die hier aufgeführten Thesen aus der Psychologie stützen meine Integration des TransfersZSE: Transfer ist nicht als separates Phänomen des Zweitspracherwerbs anzusehen, das dort unter nicht näher begründbaren Bedingungen auftritt, sondern als allgemeiner Bestandteil kognitiven Lernens, der beim ZSE spezifische Ausprägungen hat, dort jedoch grundsätzlich den gleichen Regularitäten wie beim ESE oder beim Lernen überhaupt unterliegt.

Diese Zusammenhänge legen es nahe, auch den Spracherwerb insgesamt integriert zu betrachten und nicht als von anderen Lernarten völlig unterschiedlichen Vorgang zu begreifen: Er ist vielmehr als spezifische Form kognitiver menschlicher Tätigkeit anzusehen, die grundsätzlich auf ähnlichen Mechanismen basiert und ähnliche Strukturen aufweist wie andere Formen menschlichen Lernens und menschlicher Erkenntnis. Der Lerner wendet nach dieser Theorie beim ZSE universelle kognitive Strategien an, die unter anderem die Anwendung bereits erworbenen Wissens in neuen Kontexten - den Transfer ${ }_{a}$ - beinhalten. Diese allgemeinen Charakteristika menschlichen Lernens weisen dabei spezifische Ausprägungen wie etwa den TransferzSE auf.

\section{Literaturnachweis}

Adiv, Ellen: Language Learning Strategies: The Relationship between L1 Operating Principles and Language Transfer in L2 Development. In: Andersen, Roger W. (Hrsg.): Second Languages. A Cross-linguistic Perspective. Rowley, Mass.: Newbury House, 1984, 125-142.

Andersen, Roger W.: Transfer to Somewhere. In: Gass, S. M. / Selinker, L. (Hrsg.): Language Transfer in Language Learning. Rowley, Mass.: Newbury House, 1983, 177-201.

Beardsmore, Hugo Baetens: Bilingualism: Basic Principles. Second edition. San Diego: College-Hill, 1986.

Clahsen, Harald / Meisel, Jürgen Michael / Pienemann, Manfred: Deutsch als Zweitsprache. Der Spracherwerb ausländischer Arbeiter. Tübingen: Narr, 1983.

Clyne, Michael: Forschungsbericht Sprachkontakt. Untersuchungsergebnisse und praktische Probleme. Kronberg/Ts.: Scriptor, 1975.Coseriu, E.: Probleme der strukturellen Semantik. Tübingen: Narr, 1973. 
Czochralski, Jan A.: Ausgewählte Probleme der Strukturinterferenz. In: Wissen-Fehler schaftliche Zeitschrift der Humboldt-Universität zu Berlin [Gesellschafts- und ! sprachwissenschaftliche Reihe] 22 (1973), 191-194.

Drever, James / Fröhlich, Werner D.: Wörterbuch zur Psychologie. 8., völlig neu bearbeitete Auflage. München: dtv, 1974.

Dulay, Heidi C. / Burt, Marina K. / Krashen, Stephen D.: Language Two. Oxford: Univ., 1982.

Eckman, Fred R.: Markedness and the Contrastive Analysis Hypothesis. In: Language Learning 27;1 (1977), 315-330.

Gagné, R. M.: Die Bedingungen des menschlichen Lernens. Hannover: Schroedel, 1969.

Haugen, Einar: The Norwegian Language in America: a Study in Bilingual Behavior. Philadelphia: Univ. of Pennsylvania, 1953.

ders.: Bilingualism in the Americas: A Bibliography and Research Guide. Alabama: Univ., 1956.

Jansen, Bert / Lallemann, Josien / Muysken, Pieter: The Alternation Hypothesis: Acquisition of Dutch Word Order by Turkish and Moroccan Foreign Workers. In: Language Learning 31,2 (1981), 315-336.

Juhász, Janós: Probleme der Interferenz. München: Hueber, 1970.

ders.: Synchronie - Diachronie und Interferenz - Integration. In: Zeitschrift für Germanistik 3,2 (1982), 443-444.

ders.: Die sprachliche Norm. Budapest: Univ., 1985.

Keim, Inken: Untersuchungen zum Deutsch türkischer Arbeiter. Tübingen: Narr, 1984.

Kellerman, Eric: Now You See It, Now You Don't. In: Gass, S. M. / Selinker, L. (Hrsg.): Language Transfer in Language Learning. Rowley, Mass.: Newbury House, 1983, 112-134.

LePage, R. B.: Kommentar zu Hasselmo. In: Kelly, L. G. (Hrsg.): Description and Measurement of Bilingualism: An International Seminar, University of Moncton, June 6-14, 1967. Toronto: Univ., 1969, 142-146.

Mackey, William Francis: Bilinguisme et contact des langues. Paris: Klincksieck, 1976. Mayerthaler, Willi: Morphologische Natürlichkeit. Wiesbaden: Athenaion, 1981.

Oksaar, Els (1979): Models of Competence in Bilingual Interaction. In: Mackey, W. F. I Ornstein, J. (Hrsg.): Sociolinguistic Studies in Language Contact. The Hague, Paris, New York: Mouton, 1979, 99-113.

Schiefele, Hans / Krapp, Andreas: Handlexikon zur Pädagogischen Psychologie. München: Ehrenwirth, 1981.

Weinreich, Uriel: Languages in Contact. TheHague: Mouton, 1963. 
Wode, Henning (1985): Zweitsprachenerwerbsforschung im Rückblick. In:Fehler Eppeneder, Ralf (Hrsg.): Lernersprache. Thesen zum Erwerb einer Fremd- ! sprache. München: Goethe-Institut / Kemmler \& Hoch, 1985, 7-66.

ders.: Einführung in die Psycholinguistik: Theorien, Methoden, Ergebnisse. nicht Ismaning: Hueber, 1988.

ert.

Zobl, H.: The Formal and Developmental Selectivity of L1 Influence on L2 Acquisition.

In: Language Learning 30,1 (1980), 43-57. 
${ }^{3}$ in eine an- | Elementen | eine ande- $\mid$ Sprache ${ }^{\circ}$

${ }^{3}$ dere, die zu | einer Sprache| re,die dort| unter dem $^{\circ}$

${ }^{3}$ einem Norm- | in einer | zur Einhal-| Einfluß ${ }^{\circ}$

${ }^{3}$ verstoß führt| anderen | tung der | einer

o 3 , Norm führt anderen o

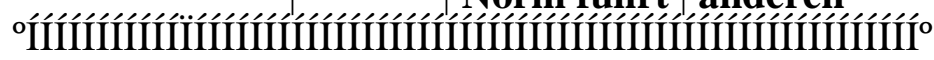

\title{
${ }^{\circ}$ HAUGEN ${ }^{3}$ transfer | interference |
}

${ }^{\circ}(1953 ; 1956)^{3}$ | (borrowing) |

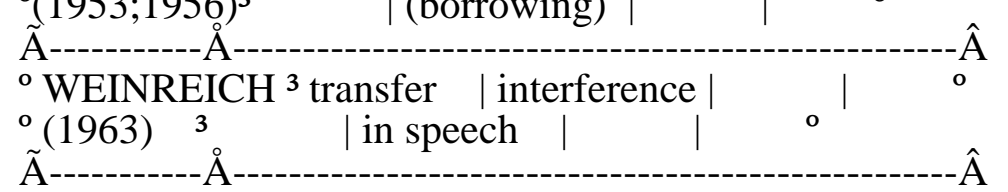

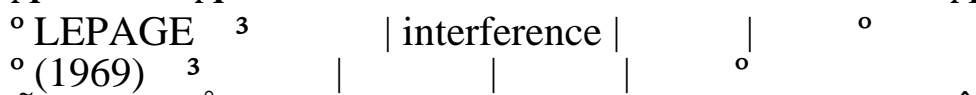

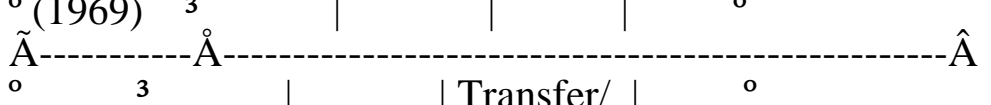

\begin{tabular}{ll|ll} 
o JUHÁSZ 3 & Interferenz | positive & & \\
$\circ$
\end{tabular}

${ }^{\circ}(1970 ; 1982)^{3} \quad|\quad|$ Interferenz| ${ }^{\circ}{ }^{0}$

${ }^{\circ}$ CZOCHRALSKI $^{3}$ | | |nterferenz | | ${ }^{3}$ | Inter- o

${ }^{\circ} \tilde{A}(1973) \quad{ }^{3} \quad|\quad| \quad \mid$ ferenz ${ }^{\circ}$

${ }^{0}(1975) \quad 3 \quad 2 \quad|\quad| \quad \mid$

${ }^{\circ}$ MACKEY ${ }^{3}$ transfer | interférence | o | o

$\tilde{\mathrm{A}}$----------- $\mathrm{A}$-----------------------------------------------------Â

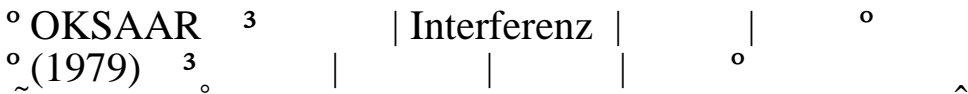

$\tilde{\mathrm{A}}-----------\mathrm{A}$ A-------------------------------------------------ÂA

${ }^{\circ}$ DULAY/BURT $/{ }^{3}$ negative |(interlingual | |(inter- ${ }^{\circ}$

${ }^{\circ}$ KRASHEN ${ }^{3}$ transfer | errors) | positive | lingual ${ }^{\circ}$

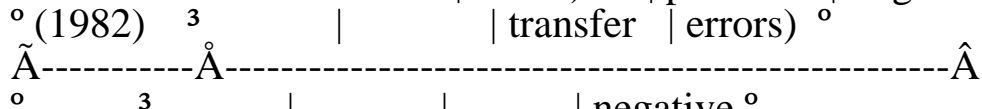

${ }^{\circ}$ BEARDSMORE ${ }^{3} \quad$ (negative) transfer | $^{\mid}$negative $^{\circ}$ | inter- ${ }^{\circ}$

$\left.{ }^{\circ}(1986)\right)^{3}$

ĖÍÍÍÍÍÍÍÍÍÍøIIIÍÍÍÍÍÍÍÍÍÍÍÍÍÍÍÍÍÍÍÍÍÍÍÍÍÍÍÍÍÍÍÍÍÍÍÍÍÍÍÍÍÍÍÍÍÍ1⁄4

Abb. 1:

Überblick über die Begrifflichkeit in einigen Ansätzen aus der Interferenzforschung

\section{Simplifizierung}

\author{
der Struktur \\ der Struktur \\ des Systems \\ der Elemente \\ = Generalisierung: <-------> = Simplifizierung \\ im engeren Sinne:
}


Verallgemeinerung

bzgl. der Verwendung
Fehler

Textm

arke nicht defini ert.

\begin{tabular}{ccccc} 
bekannter & bekannter & \multicolumn{2}{c}{ von } & von \\
Strukturmuster & Einheiten & \multicolumn{2}{c}{ Strukturmustern } & Einheiten \\
-> Simpl. & -> Simpl. & -> Simpl. & -> Simpl. \\
des & des & der & der & \\
Regel- & Einheiten- & Regeln & Einheiten \\
inventars & inventars & & &
\end{tabular}

Abb. 2: $\quad$ Hierarchie der Lernstrategien im Zweitspracherwerb 


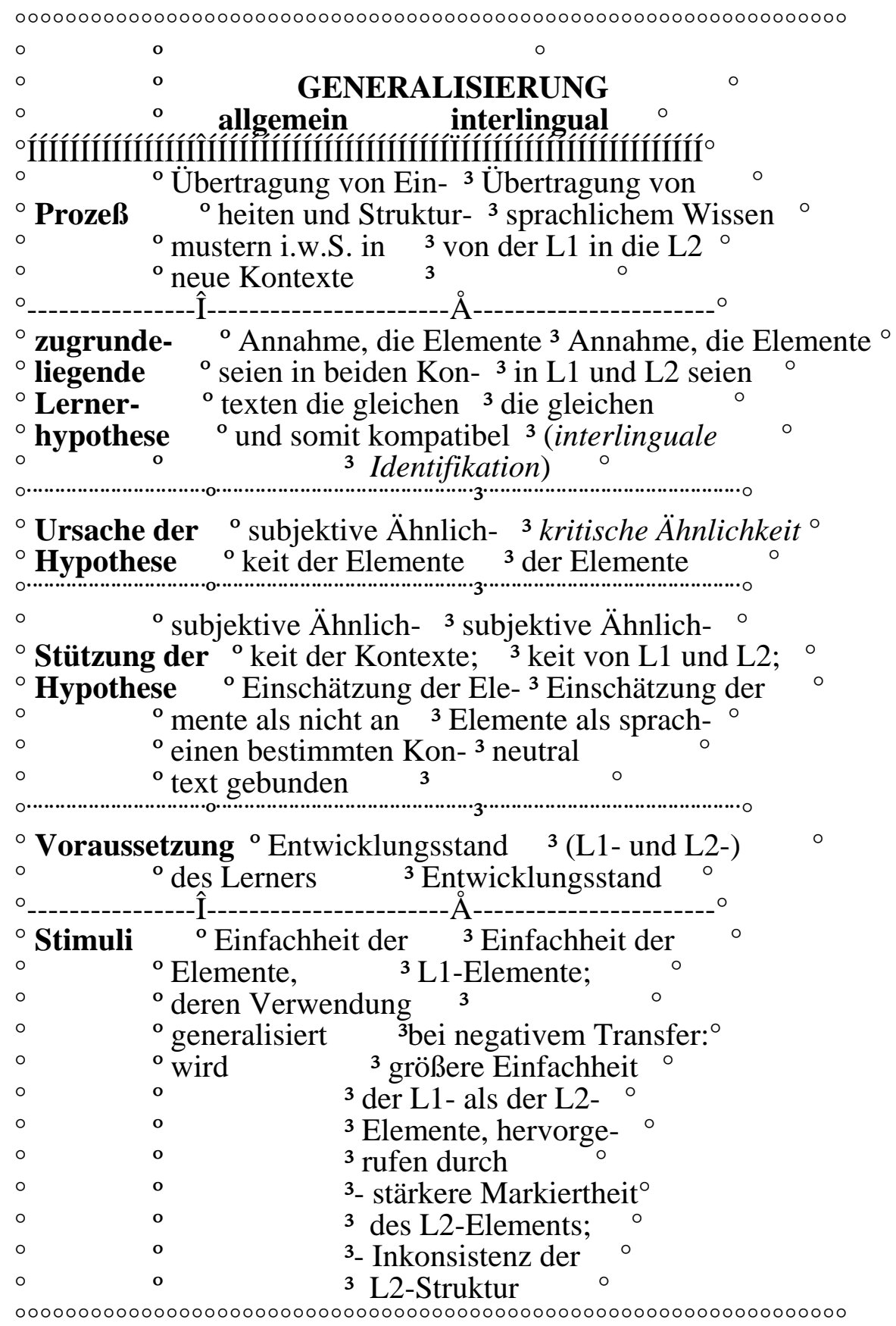

Abb.3: Charakteristika der Generalisierung und ihre interlingualen Ausprägungen 
Fehler

Textm

arke nicht defini ert. 